\title{
De la recherche à la qualité des soins
}

\section{Marc-Yvan Côté}

\section{ADRESSE}

M.Y. Côté : Ministre de la Santé et des Services sociaux du Québec. Gouvernement du Québec, Québec, Canada.

\section{TIRÉS A PART}

M.Y. Côté. es nombreuses recherches effectuées dans le domaine des sciences biomédicales, au cours des dernières décennies, ont favorisé une espérance de longévité accrue et une diminution sensible de la morbidité. Les résultats spectaculaires obtenus en recherche laissent entrevoir des progrès allant à la limite du possible. Grâce aux nombreuses innovations technologiques dont on bénéficie à l'heure actuelle, on peut observer une accélération du rythme des découvertes, en particulier en biologie cellulaire et moléculaire, en sciences neurologiques et en génétique.

La diffusion des connaissances et des techniques joue un rôle clé. Au niveau international, un nombre croissant de revues spécialisées de haut niveau, dont le contenu est supervisé par des comités de pairs, en constitue l'outil essentiel.

Je suis très fier, comme ministre de la Santé et des Services sociaux du Québec et comme francophone, de m'associer aux artisans de médecine/sciences pour célébrer le cinquième anniversaire de cette revue, qui bénéficie déjà des plus hautes reconnaissances dans le milieu scientifique. Notre contribution à médecine/sciences s'inscrit dans la foulée de nos efforts pour développer la recherche biomédicale au Québec. Le chemin parcouru depuis la fondation du Fonds de la recherche en santé du Québec, il y a un quart de siècle, mérite d'être souligné. Nous comptons maintenant sur une communauté de chercheurs dont la valeur est reconnue. $\mathrm{La}$ recherche biomédicale apparaît comme un élément essentiel à l'amélioration des soins, mais articulée dans un ensemble plus vaste. En d'autres termes, nous cherchons à orienter la recherche de sorte qu'elle puisse contribuer à la qualité des soins, entendue dans un sens plus large que la seule performance technique. Permettez-moi de tenter d'illustrer les grandes lignes de cette orientation. Le fondamental et le clinique. Fondamentale, appliquée, les deux faces de la réalité de la science sont inséparables et s'alimentent mutuellement. En milieu clinique, seule la méthode sépare les aspects fondamentaux et appliqués. La technologie permet d'intégrer de plus en plus rapidement les résultats de la recherche à la solution de problèmes cliniques. En retour, ces problèmes provoquent un développement plus rapide de modèles et de connaissances. On comprend que ces interactions tendent à rapprocher les chercheurs de divers horizons disciplinaires. En concentrant le développement de la recherche dans les centres hospitaliers, le Fonds de la recherche en santé du Québec a voulu favoriser l'application clinique des découvertes fondamentales et ainsi améliorer plus rapidement la qualité des soins aux patients.

Cette approche pragmatique a porté fruit ; des découvertes québécoises sont maintenant utilisées partout, 
notamment dans le dépistage de l'hypothyroïdie congénitale et dans le traitement du cancer de la prostate (LHRH). Mentionnons également la découverte de l'antigène carcinoembryonnaire (ACE) qui permet de suivre l'évolution de plusieurs cancers et celle de l'ANF (atrial natriuretic factor) dans l'hypertension.

Il faut cependant dépasser cette fusion du fondamental et du clinique pour tenter d'équilibrer toutes les approches du développement des connaissances et de l'amélioration des soins. Seule est capable d'une telle souplesse une structure où l'on favorise à la fois les interactions entre les méthodes et les disciplines, la formation des chercheurs et l'application des résultats.

Nous devons aussi favoriser l'émergence de disciplines et d'approches nouvelles, relevant non seulement des sciences de la santé mais encore des sciences sociales. Il importe aussi d'accentuer la collaboration entre les secteurs de la santé et de l'industrie. A mon sens, ce qui constitue l'originalité du système québécois et qui en fera de plus en plus la force, c'est l'intégration des aspects médicaux et sociaux.

Le médical et le social. En effet, au Québec, un ministère unique est responsable des services de santé et des services sociaux. Ce jumelage, certes relatif et perfectible, se répercute nécessairement sur l'orientation générale des politiques, en particulier celle de la recherche. L'insistance du Fonds de la recherche en santé du Québec sur les aspects multidisciplinaires et multiméthodologiques $y$ trouve sa source, ce qui n'est pas sans rappeler la philosophie que l'Organisation mondiale de la santé a développée dans ses documents de réflexion.

J'aimerais souligner un aspect de la recherche scientifique qui a trouvé chez nous un terrain fertile. De grandes enquêtes épidémiologiques, comme celle toute récente de "SantéQuébec ", ont permis de dresser un bilan de santé de la population. Les connaissances ainsi obtenues ont permis d'appliquer des mesures précises dans nos réseaux, comme par exemple dans nos départements de santé communautaire. Il s'agit au fond d'une démarche assez classique. $\mathrm{m} / \mathrm{s} n^{\circ} 3$ vol. 6, mars 90

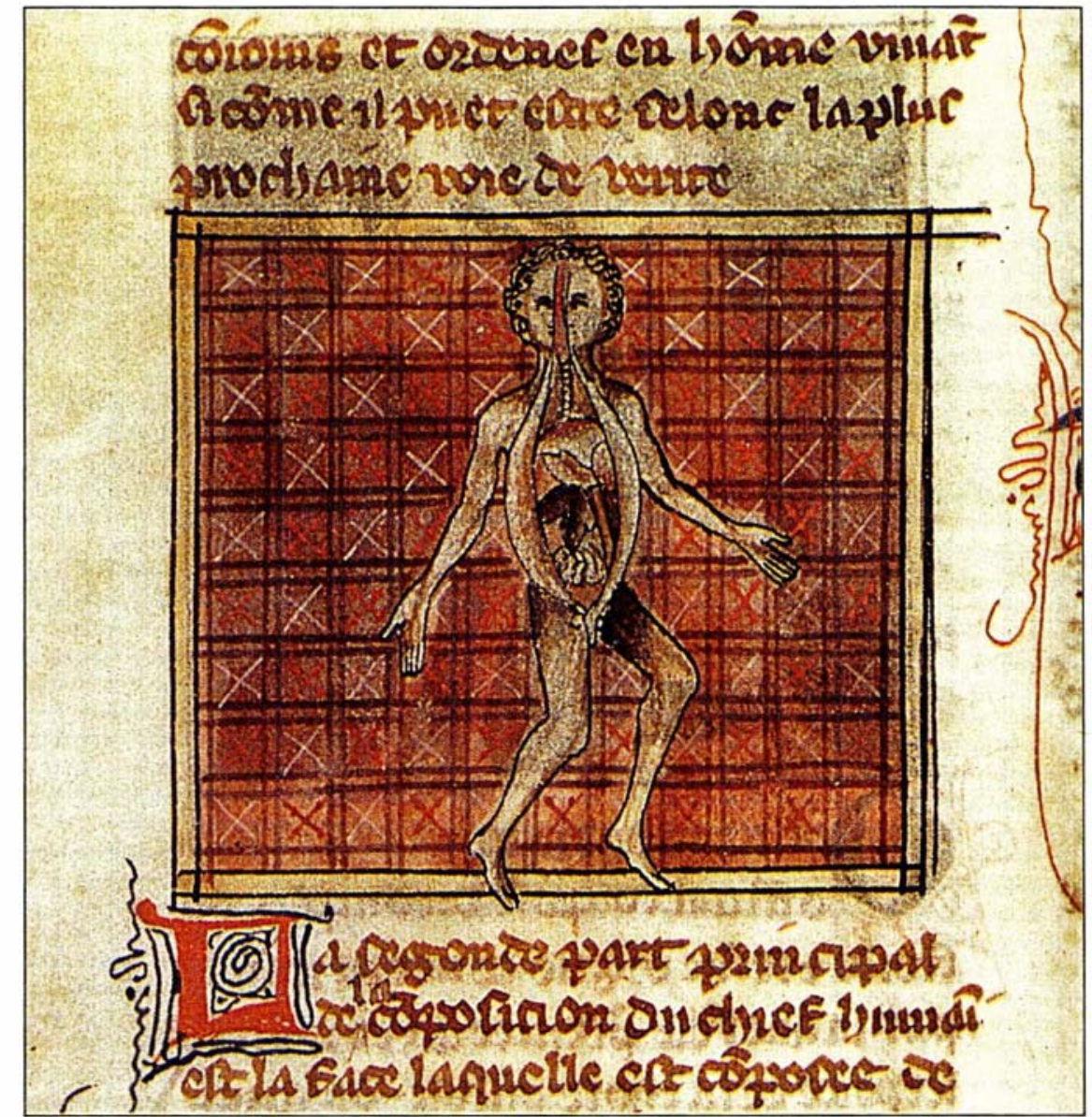

Manuscrit français de 1314 de Henri de Mondeville. Ivoir légende de la page 193). (Ms Fr. 2030, chirurgien de Mondeville, Bibliothèque Nationale de Paris).

Beaucoup de recherches en cancérologie sont issues d'études épidémiologiques. Ce type de recherche entretient donc des liens étroits avec la recherche médicale proprement dite. Ainsi, l'identification de certains facteurs héréditaires et la constitution de banques de données comme celles du Centre interuniversitaire de recherches sur les populations (SOREP) ont permis d'orienter les recherches et les interventions d'une équipe multicentrique, le Réseau de médecine génétique.

Une étude réalisée par l'École des hautes études commerciales de Montréal a conclu à la rentabilité des applications de la recherche par le Réseau de médecine génétique du Québec. On voit donc que l'alliance entre les disciplines des sciences bio- médicales et des sciences sociales peut avoir des conséquences bénéfiques sur les thérapies et en dernière analyse sur la qualité des soins prodigués aux malades.

Fort d'expériences comme celle-ci, le ministère s'est engagé résolument sur la voie de l'intégration disciplinaire. Les problèmes de santé et les problèmes sociaux sont intimement liés. Recherche fondamentale, clinique ou épidémiologique, recherche évaluative, organisationnelle et opérationnelle ou recherche sociale appliquée, toutes ont un rôle à jouer dans l'amélioration de la qualité des soins de santé. Pour obtenir la synergie souhaitée, nous devons maintenant viser un développement plus harmonieux de chacun des éléments de cet ensemble 